\title{
Enhancing pediatric adverse drug reaction documentation in the electronic medical record
}

Authors: Emma M. Tillman, Pharm.D., Ph.D. ${ }^{1}$ Sarah L. Suppes, Pharm.D. ${ }^{2}$ Keith

Feldman, Ph.D. ${ }^{3}$ Jennifer L. Goldman, M.D., M.S. ${ }^{3}$

Affiliations:

1. Division of Clinical Pharmacology, Indiana University School of Medicine

2. Department of Pharmacy, Children's Mercy Hospital

3. Department of Pediatrics, Children's Mercy Hospital and the University of MissouriKansas City

\section{Keywords:}

Pediatrics, pharmacovigilance, Drug-Related Side Effects and Adverse Reactions, electronic health record, patient safety

\section{Corresponding author:}

Emma M. Tillman, Pharm.D., Ph.D., Associate Research Professor, Division of Clinical Pharmacology Indiana University School of Medicine 950 West Walnut Street, Room 478, Indianapolis, IN 46202

317.274.2797

emtillma@iu.edu

Data sharing: Readers can contact the corresponding author by email for queries about the data; however the authors cannot directly share the data per institutional review board.

Word count for the body of the manuscript: 2,138

Number of figures and tables: 4 (Tables $1-3$, and supplementary table 1 )

Number of References: 21

This is the author's manuscript of the article published in final edited form as:

Tillman, E. M., Suppes, S. L., Feldman, K., \& Goldman, J. L. (2021). Enhancing pediatric adverse drug reaction documentation in the electronic medical record. The Journal of Clinical Pharmacology, 61(2), 181-186. https://doi.org/10.1002/jcph.1717 


\section{Abstract}

Adverse drug reactions (ADRs) often go unreported or are inaccurately documented in the electronic medical recorded (EMR), even when they are severe and life-threatening. Incomplete reporting can lead to future prescribing challenges and reoccurrences of ADRs. The aim of this study was to evaluate the documentation of ADRs within the EMR and determine specific factors associated with appropriate and timely ADR documentation. Retrospective data were collected from a pediatric hospital system ADR reports from October 2010 - November 2018. Data included implicated medication, type and severity of reaction, treatment location, the presence or absence of ADR documentation into the EMR alert profile within 24 hours of the ADR hospital or clinic encounter discharge, ADR identification method, and the presence or absence of pharmacovigilance oversight at the facility where the ADR was treated. A linear regression model was applied to identify factors contributing to optimal ADR documentation. A total of 3,065 ADRs requiring medical care were identified. Of these $961(31 \%)$ of the ADRs did not have appropriate documentation added to the EMR alert profile prior to discharge. ADRs were documented in the EMR $87 \%$ of the time with the presence of pharmacovigilance oversight and only $61 \%$ without prospective pharmacovigilance $(p<0.01)$. Severity of ADR was not a predictor of ADR documentation in the EMR, yet the implicated medication and location of treatment did impact reporting. An active pharmacovigilance service significantly improved pediatric ADR documentation. Further work is needed to assure timely, accurate ADR documentation. 


\section{Background}

Adverse drug reactions (ADRs) are an unintended reaction to a medicine given at a normal drug dose. ${ }^{1}$ ADRs occur frequently however they are notoriously underreported within health care facilities. ${ }^{2}$ Accurate ADR documentation requires a medical provider to enter the information into the medical record at the point of reaction occurrence. Access to the correct information regarding a patient's history of ADRs is critical for healthcare providers to safely prescribe medication and prevent future ADRs. ${ }^{3-6}$

Despite the importance of complete and accurate documentation, providers often fail to both gather the critical information about ADRs and appropriately document the information in the electronic medical record (EMR). ${ }^{3-5}$ For example, a provider may document "allergy to penicillin" in the EMR, yet, this documentation alone does not clarify if the patient developed life threating anaphylaxis or a common expected side effect such as diarrhea. Without detailed information, a provider is unable to determine which medications are safe or should be avoided in the future. Even when a provider gathers relevant information, many ADRs that are noted in the patient's history often fail to be added to the patient's ADR alert profile. Thus, computer decision support alerts will not warn the prescriber of the patient's history of an ADR and future prescribing clinicians may be unaware of the ADR. Poor documentation of ADRs has two potential undesired results: 1) risk of re-exposing patients to medications which have caused a previous $A D R$ and 2) avoiding a first line therapy unnecessarily based on an inaccurately documented ADR, thus providing suboptimal care. 
Evidence has shown that multidisciplinary teams involving both clinicians, including pharmacists have proven to be effective in enhancing ADR detection and reporting in clinical care. ${ }^{7,8}$ In October 2010, a drug safety service (DSS) was developed at our institution to address low ADR identification and lack of standardized ADR documentation. ${ }^{9,} 10$ The aim of this study was to evaluate the documentation of ADRs within the EMR and determine specific factors associated with appropriate ADR documentation.

Materials and Methods

Study Design

Children's Mercy Hospital (CMH) system includes a 354-bed free-standing nonprofit academic pediatric hospital, a regional hospital campus, and four regional urgent care facilities. Comprehensive primary and tertiary care in 40 pediatric subspecialties is provided to a 5 -state, 100-county region. In October 2010, a drug safety service (DSS) was developed to perform hospital-wide pharmacovigilance. A dedicated DSS pediatric clinical pharmacist collects a standardized set of data regarding ADRs by reviewing EMR data, $\left(\right.$ Cerner $^{\circledR}$, Kansas City, MO) interviewing the caregiver/child, and/or obtaining records from outside primary care physicians and pharmacies to determine the type and severity of the reported ADR. ADRs are systematically classified and documented in the EMR by type (hypersensitivity, side effect, precaution, religious or personal preference) and severity (mild, moderate, severe).

The DSS primarily provides active pharmacovigilance to the main academic hospital inpatient units Monday-Friday identifying ADRs that have occurred in 
hospitalized patients. ADRs are identified by several hospital specific daily reports (Table 1) as well as utilizing International Classification of Disease (ICD) codes (Supplementary Table). Although a primary focus of the DSS is to prospectively identify and document ADRs in hospitalized children, the service also captures and documents ADRs throughout the entire medical system via generated ADR reports. All identified ADRs are reviewed by the DSS pharmacist and entered into the EMR using the standardized ADR type and severity classifications, however the reviews of ADRs outside the main hospital most commonly occur after the patient encounter has occurred.

Quarterly, the DSS reports to the hospital wide Pharmacy and Therapeutics (P\&T) committee, providing information on ADRs identified throughout the medical system. The purpose of these reports is to: 1) update P\&T on ADR numbers, 2) identify any new or currently unrecognized ADR patterns, and 3) review the location and documentation practices of ADRs. For this study, we performed a retrospective review of data collected from the DSS generated P\&T ADR reports. Once institutional review board approval was obtained, data relating to ADRs were collected from October 1 , 2010 until November 30, 2018. These data included 1) ADR details: implicated medication, ADR phenotype (e.g. rash, anaphylaxis), reaction severity (mild, moderate, severe), reaction symptom type (allergy or side effect), and 2) information on ADR encounter: medical team treating the ADR (i.e. inpatient, outpatient, emergency department), documentation of the ADR within the EMR within 24 hours of admission, and presence or absence of the DSS based on ADR location. 
ADRs were excluded for the following reasons: unknown severity (26), ADR was treated at home and not detected until a later date (88), unknown treatment location (42) unknown ADR location (2), mechanism of ADR was precautionary, religious/preference or unknown (17), missing ADR reaction type (1), missing data on how ADR was identified (19).

Both ADR reaction and medication class were generalized into categories to facilitate analysis. In both cases, if individual ADRs or medications did not easily fit into a category or occurred less than $1 \%$ of the time, it was assigned to the "other" category. Analysis

In line with our primary objective to evaluate the documentation of ADRs within the EMR and determine specific factors associated with appropriate ADR documentation, descriptive statistics were used to evaluate variance in the incidence of ADR documentation status at $>24$ hours following reaction occurrence. For each of the study variables detailed above, unadjusted statistical comparisons were performed against ADR documentation status using Fisher's Exact tests, to account for low prevalence cells in rare drug classes or reaction types. Due to the factorial number of cells that must be evaluated, factors with over 10 levels were evaluated using 10,000 iteration Monte Carlo simulation of the Fisher's test.

L1- regularized multiple logistic regression was used to evaluate the magnitude and directionality of the association of each covariate with ADR documentation status adjusted for potential confounders identified in the univariate analyses prior. Of note, due to the highly interconnected nature of where the medication was administered and the medical treating team, medication location was not included in the final regression to 
protect against multicollinearity and improve the stability of the resulting coefficients. Additionally, rather than report statistical significance at a single arbitrary cutoff, this work follows recommendations recent work by Wasserstein et al. ${ }^{11}$ We report $p$-values for all covariates, and those that reach significance at a 95\% confidence levels.

\section{Results}

During the study period, 3,260 ADRs were identified. After exclusions, a total of 3,065 ADRs were included in the analysis. Of these 961 (30\%) did not have appropriate documentation added to the EMR alert profile within 24 hours of the ADR hospital or clinic encounter discharge (Table 2). Based on ADR severity, mild ADRs were undocumented in $18 \%$ of cases, moderate in $34 \%$ of cases and severe in $15 \%$ of cases. The most common implicated medication class was antimicrobials with a total of 1,852 ADRs detected; 623 (33\%) were undocumented in the EMR at time of occurrence. The most common detected ADRs were rash $(1064 ; 35 \%)$ and hives $(563 ; 18 \%)$ which were undocumented during encounter $42 \%$ and $34 \%$ of the time, respectively. The mechanism of ADR was classified as allergy/hypersensitivity 2,108 (69\%) of the time or side effect in 957 (31\%) cases. Allergy/hypersensitivity reactions were undocumented $36 \%$ of the time and side effects were documented $21 \%$ of the time.

The majority of the ADRs occurred after the medication was given at home (1819; $59 \%)$ followed by inpatient $(764 ; 25 \%)$, outpatient clinics $(294 ; 10 \%)$, and the emergency department (ED) (188; 6\%). ADRs were most often treated during an inpatient admission (1364; 44\%), and in the ED (942; 31\%). ADRs treated in the inpatient setting had the highest rate of documentation (84\%) and the ED had the lowest documentation rate (53\%). 
As previously mentioned, the DSS only provides active pharmacovigilance to the main academic hospital and not at the outpatient clinics or the ED. The presence of a prospective pharmacovigilance service was a significant predictor of ADR documentation where ADRs were documented in the EMR $87 \%$ of the time and only $60 \%$ without prospective pharmacovigilance. Retrospective identification of ADRs occurred $37 \%$ of the time by newly entered ADR, $22 \%$ ICD codes, $19 \%$ DSS, $15 \%$ discharge ADR reports, and $<3 \%$ of the time for clinical pharmacology consult, ADR referrals, sedation reports, laboratory triggers, and diphenhydramine triggers.

Multiple logistic regression identified several factors that were associated with ADR documentation in the EMR (Table 3). Because antimicrobials were the most common implicated medication class, it served as the medication comparison in the linear regression analysis. Compared to antimicrobials, both contrast and opioids were more likely to be documented whereas anticonvulsants, topical medications, and the multiple potential medications group were less likely to be documented. Compared to hives, red man's syndrome and anaphylaxis were less likely to go undocumented. ADR type was not associated with a difference in ADR documentation. ADR treatment location was identified as an important factor associated with ADR documentation with outpatient and ED locations twice as likely to not document an ADR as compared to the inpatient setting.

\section{Discussion}

We report ADR documentation rates in the EMR within a pediatric hospital system. Specifically, our results highlight three key findings: 1) more severe ADRs does not assure timely documentation in the EMR profile, 2) the location where an ADR was 
treated was associated with different documentation rates, and 3) the presence of a pharmacovigilance service greatly increased successful documentation of ADRs within the EMR.

In our study, severity did not directly correlate with documentation. Severe and mild ADRs had similar documentation rates but these rates were lower than documentation rates for moderate ADRs. It is not unexpected that mild and less clinically significant ADRs resulted in lower documentation as this has been previously described, ${ }^{12}$ however it was unexpected that $15 \%$ of severe ADRs were not immediately documented in the ADR alert bar. ${ }^{13}$ Accurate documentation of the specific ADR reaction and classification in the EMR is essential to optimize prescribing. Importantly, documentation of severe ADRs is critical in preventing the use of an implicated medication that caused a severe ADR at a later date leading to increased morbidity and potential mortality. ${ }^{4}$ Similarly, documentation of mild ADRs can be instrumental to prescribers so as not to avoid a medication that could potentially be prescribed if benefit outweighs the risk of the mild reaction.

The location of ADR treatment was a significant factor affecting documentation. Treatment of ADRs in an outpatient clinic or ED was associated with a lower documentation rate compared to treatment as part of an inpatient admission. Even when children experienced an ADR and sought medical care in the outpatient or ED setting for the ADR, the reaction was undocumented $45 \%$ of the time. Although we note that many of these ADRs occurred at home, these were documented after receiving care for the ADR and not historic reports of a vague history of an ADR. The lack of accurate documentation in the ED is not unique to our institution. ${ }^{5,14}$ The discordance of 
ADR reporting rates in the ED and outpatient settings compared to inpatient documentation at our institution is likely due to active pharmacovigilance oversite in the inpatient setting.

We report the presence of a pharmacovigilance service greatly increased timely documentation of ADRs within the medical record. This is consistent with reports from other centers, after incorporation of a prospective pharmacovigilance service. . $^{3,7,8,15-19}$ While the increased detection of ADRs after incorporation of a prospective pharmacovigilance program is well documented, these programs are unfortunately not the standard in all hospitals. Developing standardized EMR alerts or reports to detect ADRs requires time and infrastructure..$^{3,6,16,20,21}$ Additionally personnel, typically a clinical pharmacist, is needed to evaluate automated EMR reports and investigate potential ADRs. ${ }^{17,18}$

While personnel, EMR tools, and usability are very important, education and a positive culture of ADR reporting can be invaluable in increasing ADR reporting rates. ${ }^{19}$, ${ }^{20}$ Empowering nurses, physicians, pharmacists, and other members of the healthcare team to take ownership in reporting ADRs can greatly improve ADR reporting. ${ }^{3,21}$ Even with a robust pharmacovigilance program, opportunities to increase ADR identification and documentation still remain. Unless a program is functioning 24 hours a day, 7 days a week, ADRs will be missed. This suggests that this may be a larger heath-system issue that needs to be addressed with improved education, user friendly EMR, and potentially increased laboratory or clinical triggers that prompt the clinician to document. Our study did have limitations. This study was a retrospective review at a single institution, limiting the generalizability of our findings. This study was a natural experiment in implementation of pharmacovigilance and here we describe how ADRs were captured by a 
prospective active pharmacovigilance service and the limitations that occur when the service is not funded for $24 / 7$ coverage in all areas of the hospital and healthcare system. The pharmacovigilance program applies several laboratory triggers and methods to identify ADRs, however additional ADRs may have been missed by these methods. An additional limitation of this study that due to the large number of ADRs reported, we were unable to provide specific patient data on ADR outcomes. It is also unclear how the presence of the pharmacovigilance program at the main hospital campus influenced the documentation practices at the other medical sites. Regardless of these limitations, our results highlight that many ADRs go undocumented, regardless of severity or medication class, and further work is needed to integrate these processes into clinical care

\section{Conclusions}

Several factors can impact the rate of ADR documentation within the EMR. The identification of factors such as specific medications, ADR types, and clinical treatment settings associated with decreased ADR documenting may help to identify targeted areas and support for increased pharmacovigilance efforts. Further work is needed to assure timely, accurate ADR documentation which is essential for avoiding repeat ADRs. 


\section{References}

1. World Health Organization. Medicines: safety of medicines - adverse drug reactions. Fact sheet N 293. Updated October 2008. Available at http://whqlibdoc.who.int/hq/2002/WHO_EDM_QSM_2002.2.pdf. Accessed May 11, 2020.

2. Guner MD, Ekmekci PE. Healthcare professionals' pharmacovigilance knowledge and adverse drug reaction reporting behavior and factors determining the reporting rates. Journal of drug assessment. 2019;8(1): 13-20.

3. Hurdle JF, Weir CR, Roth B, Hoffman J, Nebeker JR. Critical gaps in the world's largest electronic medical record: Ad Hoc nursing narratives and invisible adverse drug events. AMIA Annual Symposium proceedings / AMIA Symposium AMIA Symposium. 2003: 309-312.

4. Inglis JM, Caughey GE, Smith W, Shakib S. Documentation of penicillin adverse drug reactions in electronic health records: inconsistent use of allergy and intolerance labels. Intern Med J. 2017;47(11): 1292-1297.

5. Kiechle ES, McKenna CM, Carter H, et al. Medication Allergy and Adverse Drug Reaction Documentation Discrepancies in an Urban, Academic Emergency Department. Journal of medical toxicology : official journal of the American College of Medical Toxicology. 2018;14(4): 272-277.

6. Peddie D, Small SS, Badke K, et al. Designing an Adverse Drug Event Reporting System to Prevent Unintentional Reexposures to Harmful Drugs: Study Protocol for a Multiple Methods Design. JMIR research protocols. 2016;5(3): e169.

7. Le J, Nguyen T, Law AV, Hodding J. Adverse drug reactions among children over a 10year period. Pediatrics. 2006;118(2): 555-562.

8. Prosser TR, Kamysz PL. Multidisciplinary adverse drug reaction surveillance program. American journal of hospital pharmacy. 1990;47(6): 1334-1339.

9. Goldman JL, Sullins A, Sandritter T, Leeder JS, Lowry J. Pediatric Pharmacovigilance: Enhancing Adverse Drug Reaction Reporting in a Tertiary Care Children's Hospital. Ther Innov Regul Sci. 2013;47(5): 566-571.

10. Yu D, Sheets J, Suppes S, Goldman J. Characterization of Severe Adverse Drug Reactions at a Free-Standing Children's Hospital. J Clin Pharmacol. 2019;59(12): 15691572.

11. Wasserstein RL, Schirm AL, Lazar NA. Moving to a World Beyond " $p<0.05$ ". The American Statistician. 2019;73(sup1): 1-19.

12. Sundaran S, Udayan A, Hareendranath $\mathrm{K}$, et al. Study on the Classification, Causality, Preventability and Severity of Adverse Drug Reaction Using Spontaneous Reporting System in Hospitalized Patients. Pharmacy. 2018;6(4).

13. Parameswaran Nair N, Chalmers L, Bereznicki BJ, Curtain CM, Bereznicki LR. Repeat Adverse Drug Reaction-Related Hospital Admissions in Elderly Australians: A Retrospective Study at the Royal Hobart Hospital. Drugs \& aging. 2017;34(10): 777-783.

14. Rydberg DM, Holm L, Engqvist I, et al. Adverse Drug Reactions in a Tertiary Care Emergency Medicine Ward - Prevalence, Preventability and Reporting. PLoS One. 2016;11(9): e0162948.

15. He W, Yao D, Hu Y, Dai H. Analysis of a pharmacist-led adverse drug event management model for pharmacovigilance in an academic medical center hospital in China. Therapeutics and clinical risk management. 2018;14: 2139-2147.

16. Hui C, Vaillancourt R, Bair L, Wong E, King JW. Accuracy of Adverse Drug Reaction Documentation upon Implementation of an Ambulatory Electronic Health Record System. Drugs - real world outcomes. 2016;3(2): 231-238.

17. Ekhart $C$, de Vries $T$, van Hunsel F. Psychiatric adverse drug reactions in the paediatric population. Arch Dis Child. 2020;0:1-7. 
18. Morales-Rios O, Cicero-Oneto C, Garcia-Ruiz C, et al. Descriptive study of adverse drug reactions in a tertiary care pediatric hospital in Mexico from 2014 to 2017. PLoS ONE. 2020;15(3): e0230576

19. Robelo Gomez E, Ribeiro-Vaz I, Santos CC, Herdeiro MT. Adverse drug reactions in adolescents: A review of reporting to a National Pharmacovigilance System. Expert Opin Drug Safety. 2020;DOI:10.1080/14740338.2020.1771305

20. Ramirez E, Carcas AJ, Borobia AM, et al. A pharmacovigilance program from laboratory signals for the detection and reporting of serious adverse drug reactions in hospitalized patients. Clin Pharmacol Ther. 2010;87(1): 74-86.

21. Choi SA, Kim H, Kim S, et al. Analysis of antiseizure drug-related adverse reactions from the electronic health record using the common data model. Epilepsia. 2020;61:610616.

22. Toklu $\mathrm{HZ}$, Mensah $\mathrm{E}$. Why do we need pharmacists in pharmacovigilance systems? Online journal of public health informatics. 2016;8(2): e193.

23. Morales Rios O, Jasso Gutierrez L, Talavera JO, et al. A comprehensive intervention for adverse drug reactions identification and reporting in a Pediatric Emergency Department. International journal of clinical pharmacy. 2016;38(1): 80-87.

24. Pagotto $C$, Varallo F, Mastroianni P. Impact of educational interventions on adverse drug events reporting. International journal of technology assessment in health care. 2013;29(4): 410-417.

25. Goldstein LH, Berlin M, Saliba W, Elias M, Berkovitch M. Founding an adverse drug reaction (ADR) network: a method for improving doctors spontaneous ADR reporting in a general hospital. J Clin Pharmacol. 2013;53(11): 1220-1225. 
Table 1: Drug safety service reports used to identify ADRs

\begin{tabular}{|l|l|l|}
\hline Report & Description & $\underline{\text { Percent Identification }}$ \\
\hline $\begin{array}{l}\text { Drug Safety } \\
\text { Service (DSS) }\end{array}$ & $\begin{array}{l}\text { Identified and independently documented } \\
\text { just by the DDS pharmacist }\end{array}$ & $\underline{15 \%}$ \\
\hline $\begin{array}{l}\text { Newly entered } \\
\text { ADR }\end{array}$ & $\begin{array}{l}\text { Automated daily report that pulls any ADR } \\
\text { that has newly been entered into a } \\
\text { patient's ADR profile (ED \& inpatient) }\end{array}$ & $\underline{37 \%}$ \\
\hline $\begin{array}{l}\text { Discontinued due } \\
\text { to ADR }\end{array}$ & $\begin{array}{l}\text { Automated daily report that pulls any } \\
\text { patient that has an active order on his/her } \\
\text { MAR, but the same or similar medication } \\
\text { in the ADR profile (ED \& inpatient) }\end{array}$ & $\underline{15 \%}$ \\
\hline ICD code & $\begin{array}{l}\text { Report generated through EMR utilizing } \\
\text { the below billing codes (ED, clinic, \& } \\
\text { inpatient) }\end{array}$ & $\underline{22 \%}$ \\
\hline $\begin{array}{l}\text { Diphenhydramine } \\
\text { trigger }\end{array}$ & $\begin{array}{l}\text { Any patient that has a diphenhydramine } \\
\text { order on his/her MAR }\end{array}$ & $\underline{<3 \%}$ \\
\hline ADR Referral & $\begin{array}{l}\text { Voluntary notification built within EMR, } \\
\text { sends a notification to the DSS } \\
\text { pharmacist }\end{array}$ & $\underline{<3 \%}$ \\
\hline Sedation report & $\begin{array}{l}\text { Nursing sedation documentation form, } \\
\text { question asking if ADR an occurred } \\
\text { during procedure }\end{array}$ & $\underline{<3 \%}$ \\
\hline
\end{tabular}

ADR, adverse drug reaction; DSS, drug safety service; ED, emergency department; EMR, electronic medical record; MAR, medication administration record 
Table 2: Unadjusted factors associated with ADR documentation

\begin{tabular}{|l|l|l|l|}
\hline & $\begin{array}{l}\text { Documented } \\
\text { N=2104 (\%) }\end{array}$ & $\begin{array}{l}\text { Not Documented } \\
\text { N=961 (\%) }\end{array}$ & P-value \\
\hline Severity & & & $\mathrm{p}<0.01$ \\
\hline Mild & $54(82)$ & $12(18)$ & \\
\hline Moderate & $1704(66)$ & $890(34)$ & \\
\hline Severe & $346(85)$ & $59(15)$ & \\
\hline Implicated Medication, by Class & & & $\mathrm{p}<0.01$ \\
\hline Anti-inflammatory, steroid & $44(63)$ & $25(36)$ & \\
\hline Antimicrobial & $1229(66)$ & $623(33)$ & \\
\hline Anticonvulsant & $69(61)$ & $43(39)$ & \\
\hline Antiemetic & $64(82)$ & $14(18)$ & \\
\hline Antifungal & $23(74)$ & $8(26)$ & \\
\hline Antihistamine & $39(67)$ & $19(33)$ & \\
\hline Benzodiazepine & $79(77)$ & $23(23)$ & \\
\hline Chemotherapy & $67(88)$ & $9(12)$ & \\
\hline Contrast & $55(93)$ & $4(7)$ & \\
\hline Multiple drugs & $30(52)$ & $28(48)$ & \\
\hline Opioid & $175(82)$ & $36(17)$ & \\
\hline Other & $167(65)$ & $91(35)$ & \\
\hline Sedative, anesthesia & $26(76)$ & $8(23)$ & \\
\hline Topical & $40(57)$ & $30(43)$ & \\
\hline ADR Symptom & & & \\
\hline Anaphylaxis & $235(82)$ & $50(18)$ & \\
\hline Behavioral & $98(70)$ & $42(30)$ & \\
\hline Cardiac & $34(81)$ & $8(19)$ & \\
\hline Dystonia & $36(81)$ & $8(19)$ & \\
\hline Gl & $100(79)$ & $27(21)$ & \\
\hline Hives & $366(65)$ & $197(34)$ & \\
\hline Itching & $29(69)$ & $13(30)$ & \\
\hline Liver & $28(74)$ & $10(26)$ & \\
\hline Neurologic & $77(73)$ & $28(26)$ & \\
\hline Other & $193(78)$ & $56(22)$ & \\
\hline Rash & $618(58)$ & $446(42)$ & \\
\hline Red man's syndrome & $143(92)$ & $13(8)$ & \\
\hline Respiratory distress & $44(81)$ & $10(19)$ & \\
\hline Serum sickness & $64(68)$ & $30(31)$ & \\
\hline Swelling & $39(62)$ & $23(27)$ & \\
\hline Category of ADR & & & \\
\hline Allergy/Hypersensitivity & $1352(64)$ & $756(36)$ & \\
\hline Side effect & $752(79)$ & $205(21)$ & \\
\hline Location ADR Occurred & & & \\
\hline Home & $1043(57)$ & $776(43)$ & \\
\hline Outpatient & $233(80)$ & $61(20)$ & \\
\hline Emergency department & $147(78)$ & $41(22)$ & \\
\hline Inpatient & $681(89)$ & $83(11)$ & \\
\hline ADR Treatment Location & & & \\
\hline & & & \\
\hline
\end{tabular}




\begin{tabular}{|l|l|l|l|}
\hline Outpatient & $448(59)$ & $311(41)$ & \\
\hline Emergency department & $508(53)$ & $434(46)$ & \\
\hline Inpatient & $1148(84)$ & $216(16)$ & \\
\hline Pharmacovigilance & & & $\mathrm{p}<0.01$ \\
\hline No Pharmacovigilance service & $1246(60)$ & $832(40)$ & \\
\hline Pharmacovigilance service & $857(87)$ & $129(13)$ & \\
\hline
\end{tabular}


Table 3: Adjusted odds ratio of factors associated with failure to document an ADR in the medical record

\begin{tabular}{|c|c|c|c|}
\hline \multicolumn{2}{|c|}{ ADR DOCUMENTATION FACTORS } & \multirow{2}{*}{$\begin{array}{l}\text { Odds Ratio (95\% } \\
\text { CI) } \\
\text { Reference } \\
\end{array}$} & \multirow[t]{2}{*}{ P-value } \\
\hline \multirow[t]{3}{*}{ Severity } & Mild & & \\
\hline & Moderate & $1.065(0.515-2.202)$ & 0.865 \\
\hline & Severe & $0.754(0.338-1.679)$ & 0.489 \\
\hline \multirow{14}{*}{$\begin{array}{c}\text { Implicated Medication, } \\
\text { by Class }\end{array}$} & Antimicrobial & Reference & \\
\hline & Anti-inflammatory, steroid & $1.626(0.923-2.865)$ & 0.092 \\
\hline & Anticonvulsant & $2.069(1.295-3.306)$ & 0.002 \\
\hline & Antiemetic & $0.675(0.328-1.39$ & 0.286 \\
\hline & Antifungal & $1.048(0.438-2.507)$ & 0.917 \\
\hline & Antihistamine & $1.373(0.72-2.617)$ & 0.336 \\
\hline & Benzodiazepine & $0.777(0.409-1.474)$ & 0.440 \\
\hline & Chemotherapy & $0.756(0.354-1.616)$ & 0.471 \\
\hline & Contrast & $0.172(0.059-0.499)$ & 0.001 \\
\hline & Multiple drugs & $3.109(1.707-5.663)$ & $<0.001$ \\
\hline & Opioid & $0.629(0.387-1.022)$ & 0.061 \\
\hline & Other & $1.844(1.324-2.568)$ & $<0.001$ \\
\hline & Sedative, anesthesia & $0.862(0.365-2.034)$ & 0.735 \\
\hline & Topical & $1.805(1.07-3.04)$ & 0.027 \\
\hline \multirow[t]{15}{*}{ ADR Symptom } & Hives & Reference & \\
\hline & Anaphylaxis & $0.615(0.416-0.91)$ & 0.015 \\
\hline & Behavioral & $1.323(0.693-2.601)$ & 0.383 \\
\hline & Cardiac & $0.882(0.352-2.21)$ & 0.789 \\
\hline & Dystonia & $0.913(0.33-2.527)$ & 0.861 \\
\hline & GI & $0.888(0.482-1.637)$ & 0.704 \\
\hline & Itching & $1.792(0.825-3.894)$ & 0.141 \\
\hline & Liver & $1.093(0.449-2.665)$ & 0.844 \\
\hline & Neurologic & $0.767(0.391-1.505)$ & 0.441 \\
\hline & Other & $1.109(0.669-1.84)$ & 0.688 \\
\hline & Rash & $1.342(1.076-1.675)$ & 0.009 \\
\hline & Redman & $0.481(0.23-1.009)$ & 0.053 \\
\hline & Respiratory distress & $0.648(0.293-1.434)$ & 0.285 \\
\hline & Serum sickness & $1.245(0.754-2.055)$ & 0.392 \\
\hline & Swelling & $0.989(0.537-1.82)$ & 0.971 \\
\hline \multirow[t]{2}{*}{ Category of ADR } & Allergy/Hypersensitivity & Reference & \\
\hline & Side effect & $0.863(0.56-1.329)$ & 0.504 \\
\hline \multirow[t]{3}{*}{ ADR Treatment Location } & Inpatient & Reference & \\
\hline & Outpatient & $2.116(1.58-2834)$ & $<0.001$ \\
\hline & Emergency department & $2.704(2.042-3.581)$ & $<0.001$ \\
\hline \multirow[t]{2}{*}{ Pharmacovigilance } & No & Reference & \\
\hline & Yes & $0.863(0.56-1.329)$ & $<0.001$ \\
\hline
\end{tabular}

